\title{
Visibilidad de las sedes web de los museos de Cataluña
}

\author{
Joan-Isidre Badell*, Cristòfol Rovira*
}

Resumen: El artículo presenta los resultados de un análisis de la visibilidad de todas las sedes web de museos de Cataluña entre los años 2006 y 2009. A partir de los datos obtenidos en Yahoo Site Explorer se ha podido comprobar que globalmente el número de enlaces recibidos ha aumentado en un 326,28\%. Se valora esta cifra en relación a un grupo de sedes web de referencia llegando a la conclusión que el aumento es muy importante. Por otra parte, se estudian las sedes web de los museos de Cataluña en comparación con dos grupos de control: sedes web de los principales museos de Europa y sedes web de los dominios .museum mejor posicionados en Google y Yahoo. Los resultados de la comparación indican que las mejores sedes web de los museos de Cataluña tienen un buen nivel internacional en visibilidad aunque están todavía lejos de los mejores de Europa.

Palabras clave: Visibilidad, web, museos, Cataluña, enlaces recibidos, PageRank.

\section{Visibility of Museum Web Sites of Catalonia}

Abstract: The article presents the results of an analysis of the visibility of all museums websites of Catalonia between 2006 and 2009. Based on data obtained from Yaboo Site Explorer, the study found that overall the number of links received, or backlinks, had increased by $326.28 \%$. As compared to a referenced group of websites, this represents a very important increase. Additionally, Catalan museum websites were compared to two control groups: websites of the major European museums and websites with a .museum domain that are best positioned in Google and Yaboo. The results show that the best Catalan museum websites have attained a good visibility level internationally, although they still remain at a considerable distance from the best museums in Europe.

Keywords: Visibility, website, museum, Catalonia, backlinks, PageRank.

* Departamento de Comunicación, Universidad Pompeu Fabra. Barcelona. Correo-e: joanisidre. badell@upf.edu, cristofol.rovira@upf.edu.

Recibido: 6-10-2009; 2. ${ }^{a}$ versión: 21-12-2009; aceptado: 21-1-2010. 


\section{Introducción}

El artículo presenta los resultados de un análisis de la visibilidad de las webs de museos de Cataluña, de las causas y de las posibles buenas prácticas para conseguir valores altos en este indicador. Se ha estudiado la evolución de la visibilidad de todos los sitios web de museos de Cataluña entre los años 2006 y 2009. Las URL de los museos han sido obtenidas de los directorios de museos del Ministerio de Cultura de España y de la Generalitat de Cataluña. Los datos sobre la visibilidad han sido obtenidos mayoritariamente de forma automática utilizando una herramienta de tipo rastreador conocida como DigiDocSpider (Pedraza y otros, 2008) desarrollada por el Grupo de Investigación DigiDoc de la Universidad Pompeu Fabra. Se trata de un programa informático de tipo crawler o spider que ha sido configurado para extraer datos de forma no intrusiva y abusiva de los buscadores.

Por otro lado, se han utilizado grupos de control, entre ellos el de los principales museos de Europa y el de los museos con dominio .museum, con el objetivo de establecer una comparativa de visibilidad y poder situar los resultados de los museos catalanes en el contexto internacional.

Los principales objetivos del estudio son:

- Identificar todas las webs de museos de Cataluña.

- Elaborar un ranking de visibilidad de las webs de museos de Cataluña en el año 2009.

- Comparar el ranking del 2009 con el del 2006.

- Identificar las webs que han ganado más visibilidad.

- Identificar las webs que han perdido más visibilidad.

- Comparar los datos del año 2009 con los de los grupos de control específicamente creados.

La consecución de estos objetivos nos permitirá tener los datos necesarios para profundizar en la identificación de las buenas prácticas que permitan mejorar la visibilidad de cualquier sede web de museo. Por otra parte, desde una perspectiva más general, los resultados de este estudio nos permiten avanzar en la valoración del impacto sociocultural de la dimensión digital de los museos de Cataluña.

\section{Revisión de la bibliografía}

El análisis y la evolución de sitios web de museos en nuestro país se ha realizado desde perspectivas muy diversas. La primera referencia la encontramos en 1998 con un interesante análisis realizado por López de Prado (1998) en el que se evalúan un grupo de sedes web de museos aplicando parámetros relativos al contenido y a la accesibilidad entre los cuales se encuentra el número de enlaces recibidos. Por otra parte Olsina (1999), CIFNE (2004), Monistrol (2005), Pardo 
(2008) y Burnette y otros (2009) hacen propuestas específicas de metodologías para la evaluación de sitios web de museos. En estas propuestas, los enlaces recibidos tienen una especial importancia debido a que son indicadores relativamente fiables sobre la popularidad de la sede web referenciada. También los métodos generales de evaluación de sedes web (Codina, 2000) incluyen este parámetro por ser un dato cuantitativo, relativamente objetivo.

En la bibliografía internacional abundan los estudios sobre evaluación de sedes web de museos centrados en la usabilidad (Garzotto y otros, 1998; Dyson y Morán, 2000; Cunliffe y otros, 2001; Harms y Werner, 2001; Pallas y Economilles, 2008), en el diseño centrado en el usuario (Hertzum, 1999), en los contenidos (Kravchyna y Hastings, 2002) o en su adecuación para soportar los nuevos dispositivos móviles (Mulholland y otros, 2005; Sharples y otros, 2007). En la mayoría de ellos se incluye igualmente el análisis de enlaces recibidos como parámetro de evaluación.

El análisis de enlaces se ha convertido en una práctica habitual para la evaluación de sedes web de cualquier temática. Ya sea en un contexto de estudios cibermétricos o para la valoración de su calidad. No obstante, no hemos encontrado antecedentes de estudios centrados en el análisis de enlaces de sedes web de museos. La ausencia de estos antecedentes y la importancia del parámetro analizado motivaron la realización de este estudio.

\section{Metodología}

Las URL de los museos han sido obtenidas de los directorios de museos del Ministerio de Cultura de España y de la Generalitat de Cataluña. Los datos sobre la visibilidad han sido obtenidos mayoritariamente de forma automática utilizando una herramienta de tipo rastreador conocida como DigiDocSpider desarrollada por el Grupo de Investigación DigiDoc de la Universidad Pompeu Fabra. Se trata de un programa informático de tipo crawler o spider que ha sido configurado para extraer datos de forma no intrusiva y abusiva de los buscadores.

Por otro lado, se han utilizado grupos de control, entre ellos el de los principales museos de Europa y el de los museos con dominio .museum, con el objetivo de establecer una comparativa de visibilidad y poder situar los resultados de los museos catalanes en el contexto internacional.

La visibilidad es el número de enlaces que recibe un sitio web y ofrece una idea del impacto del sitio web en Internet (Bray, 1996; Aguillo, 2000; Alonso Berrocal y otros, 2004). Los enlaces que recibe un sitio web se conocen como enlaces recibidos, entrantes, o backlinks.

En algunos casos, para calcular la visibilidad se contabilizan los enlaces entrantes que apuntan a todas las páginas del sitio web, pero en otras ocasiones solo se consideran los enlaces que apuntan a la página de inicio. Por otra parte, a veces se consideran todos los enlaces entrantes y en otras ocasiones solamente los que provienen de páginas externas y, por tanto, se eliminan los autos enlaces.

Rev. Esp. Doc. Cient., 33, 4, octubre-diciembre, 531-552, 2010. ISSN: 0210-0614. doi:10.3989/redc.2010.4.754 533 
La visibilidad está fuertemente relacionada con otro indicador cibermétrico de gran importancia, el factor de impacto (Rodríguez i Gairín, 1997; Ingwersen, 1998). El factor de impacto web intenta reproducir en la web el clásico factor de impacto de una publicación científica y se calcula dividiendo el número de enlaces entrantes que apuntan a alguna página de la web por el número total de páginas del sitio web. Algunos autores, también en el factor de impacto, eliminan los auto enlaces, no obstante, se trata de un indicador poco usado que ha recibido fuertes críticas (Noruzi, 2006).

En nuestro estudio se ha utilizado el indicador de visibilidad considerando los enlaces recibidos que apuntan a la página de inicio del web analizado. Pensamos que de esta forma se pueden comparar de manera más equitativa sitios web grandes y pequeños.

Para la elaboración del estudio se han recogido los datos sobre la visibilidad de los sitios webs de museos durante los meses de marzo, abril y mayo del 2009. La información se ha obtenido del buscador Yahoo, ya que es el único que permite obtener los datos de los enlaces recibidos separados de los auto enlaces. Otros buscadores, como por ejemplo, Google, permiten utilizar la ecuación de búsqueda link:www.sitioweb.com, pero los resultados obtenidos incluyen todos los enlaces entrantes, también los enlaces internos. Por esta razón, para la obtención de los datos de nuestro estudio, DigiDocSpider ha ejecutado en Yahoo una búsqueda para la obtención de los enlaces recibidos en las páginas de inicio de los museos analizados, exceptuando los enlaces que provienen de la propia

\section{GRÁFICO 1}

DigiDocSpider
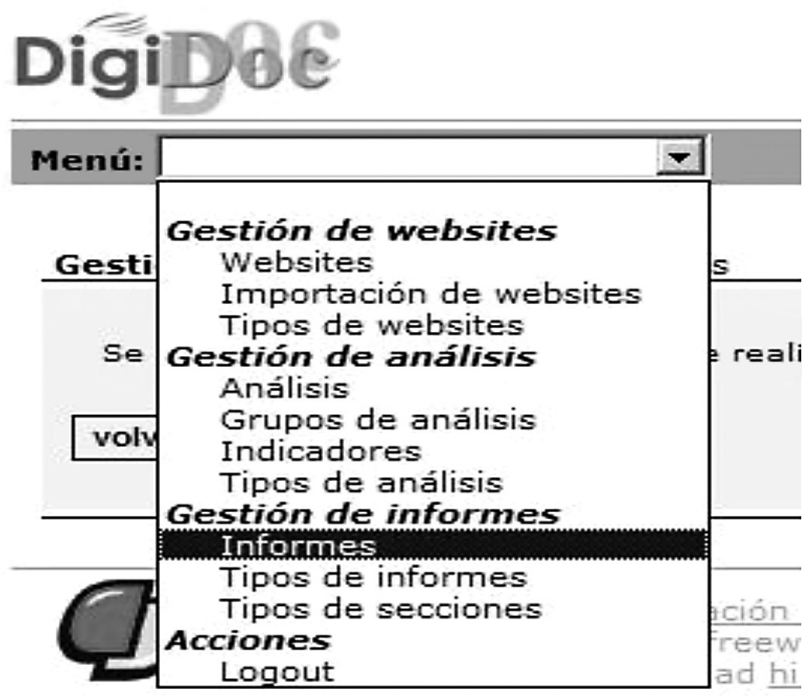

Productos de acceso libre y gratuit 
web. En concreto, ha ejecutado una búsqueda directa, sin API, en el formulario de Yahoo Site Explorer con los siguientes parámetros: «enlaces entrantes», "excepto de ese dominio" y "solo esta URL".

DigiDocSpider es un programa informático de tipo rastreador desarrollado por el Grupo de Investigación DigiDoc del Departamento de Comunicación de la Universidad Pompeu Fabra. Las capacidades del rastreador incluyen analizar las páginas web disponibles en internet extrayendo y analizando los elementos del código fuente previamente seleccionados. Así mismo, puede remitir la URL de la web analizada a servicios abiertos disponibles en línea para después compilar los resultados e incorporarlos a su informe. Esta prestación se utiliza para aplicar de forma automática validadores del tipo XHTML, accesibilidad, etc., y también para obtener datos de las webs analizadas disponibles en los buscadores, como por ejemplo, los de la visibilidad.

En total, DigiDocSpider puede recopilar de forma automática más de cien indicadores relativos a cuatro aspectos básicos de los sitios web: accesibilidad, metadatos, posicionamiento de los buscadores, visibilidad (enlaces recibidos) y calidad del código XHTML.

Los datos se han analizado desde dos perspectivas:

1. Perspectiva comparativa. Se han estudiado los datos de los sitios web de los museos de Cataluña en relación a una muestra representativa (grupo de control) de los principales museos de Europa y de los mejores con dominio .museum.

2. Perspectiva evolutiva. Se han analizado los datos obtenidos en el año 2009 con los datos publicados en estudios previos sobre los mismos sitios web en los años 2006 (Monistrol y otros, 2006).

\section{Los grupos de control}

Para los estudios comparativos, hemos creado el grupo de control de los principales museos de Europa, en concreto los quince más importantes. Este grupo de control permite comparar el estado actual de los sitios web de los principales museos catalanes con los de Europa.

Por otra parte, el grupo de control de sedes web con dominio .museum se ha formado escogiendo las veinte sedes web con un mayor número de enlaces recibidos. Los datos han sido obtenidos del ranking backlinks.info elaborado por el Grupo de Investigación DigiDoc de la Universidad Pompeu Fabra y disponible en la dirección http://www.backlinks.info.

Este grupo de control es internacional, más allá del ámbito europeo, y nos permite comparar la situación de los museos catalanes en relación a los mejores museos en visibilidad de grupo de instituciones que, previsiblemente, tienen un especial interés en su proyección en Internet ya que han gestionado y obtenido un dominio específico para museos.

Rev. Esp. Doc. Cient., 33, 4, octubre-diciembre, 531-552, 2010. ISSN: 0210-0614. doi:10.3989/redc.2010.4.754 535 


\section{Resultados}

La tabla siguiente muestra el resultado obtenido en el 2009 ordenada de mayor a menor cantidad de enlaces recibidos. Destaca en positivo el resultado obtenido por algunos museos locales o comarcales, como el de Granollers y el comarcal de Urgell, así como el diocesano de Tarragona. Estos museos locales se posicionan entre los más importantes museos catalanes que ocupan, lógicamente, los primeros puestos.

TABLA I

Ranking de los museos de Cataluña analizados (2009)

\begin{tabular}{|c|c|}
\hline Museo (ordenación por cantidad de enlaces) & Enlaces recibidos \\
\hline Museu d'Història de Catalunya & 39.618 \\
\hline Fundació Dalí & 16.972 \\
\hline Museu de Granollers & 14.486 \\
\hline Museu Nacional d'Art de Catalunya & 10.948 \\
\hline Museu Picasso & 9.700 \\
\hline Museu d'Art Contemporani de Barcelona & 6.274 \\
\hline Museu Comarcal de l'Urgell & 2.276 \\
\hline Fundació Miró & 2.229 \\
\hline Museu Diocesà de Tarragona & 2.069 \\
\hline Museu d'Història de Barcelona & 1.912 \\
\hline Museu de la Música & 1.404 \\
\hline Museu Nacional de la Ciència i la Tècnica de Catalunya & 1.148 \\
\hline Museu de l'Institut Botànic de Barcelona & 935 \\
\hline Museu de Badalona & 796 \\
\hline Museu Frederic Marès & 684 \\
\hline Museu de l'Empordà & 591 \\
\hline Museu Tèxtil i d'Indumentària & 527 \\
\hline Museu Municipal Josep Aragay (Breda) & 526 \\
\hline Museu del Joguet de Catalunya & 513 \\
\hline Museu Episcopal de Vic & 468 \\
\hline Museu del Cinema (Girona) & 392 \\
\hline Museu d'Arqueologia de Catalunya & 345 \\
\hline Museu d'Art Jaume Morera (Lleida) & 336 \\
\hline Biblioteca Museu Víctor Balaguer (Vilanova i la Geltrú) & 178 \\
\hline Museu de la Pesca de Palamós & 132 \\
\hline Museu de Ripoll & 132 \\
\hline
\end{tabular}


TABLA I (continuación)

\begin{tabular}{|c|c|}
\hline Museo (ordenación por cantidad de enlaces) & Enlaces recibidos \\
\hline Museu de Cardedeu & 107 \\
\hline Museu Geològic del Seminari de Barcelona & 101 \\
\hline Museu Salvador Vilaseca (Reus) & 101 \\
\hline Museu d'Història de Girona & 92 \\
\hline Museu d'Art Modern de Tarragona & 88 \\
\hline Museu de Gavà & 87 \\
\hline Museu Torre Balldovina & 80 \\
\hline Fundació Tàpies & 75 \\
\hline Museu de la Noguera & 61 \\
\hline Museu Diocesà d'Urgell & 53 \\
\hline Museu Municipal de Tossa de Mar & 40 \\
\hline Museu de Montserrat & 39 \\
\hline Museu Monestir de Pedralbes & 39 \\
\hline Museu de Terrassa & 37 \\
\hline Museu d'Història de Cambrils & 34 \\
\hline Museu d'Art de Girona & 22 \\
\hline Museu Molí Paperer de Capellades & 21 \\
\hline Museu Municipal de Nàutica del Masnou & 17 \\
\hline Museu del Suro de Palafrugell & 15 \\
\hline Museu de Cervera & 13 \\
\hline Museu Pau Casals & 13 \\
\hline Museu-Arxiu Municipal de Calella & 13 \\
\hline Museu del Futbol Club Barcelona & 8 \\
\hline Museu de Zoologia & 7 \\
\hline Museu d'Història de Sabadell & 6 \\
\hline Centre d'Interpretació del Parc Güell & 5 \\
\hline Museu Agrícola de Cambrils & 4 \\
\hline L'Enrajolada Casa - Museu Santacana (Martorell) & 3 \\
\hline Museu Municipal Vicenç Ros (Martorell) & 3 \\
\hline Catedral de Girona & 1 \\
\hline Museu de la Pell d'Igualada & 1 \\
\hline Museu del Vidre de Vimbodí & 1 \\
\hline Museu Deu (Vendrell) & 0 \\
\hline Museu Etnològic del Montseny & 0 \\
\hline Museu Marítim de Barcelona & 0 \\
\hline
\end{tabular}


La tabla II es una comparativa entre el estudio realizado en 2006 (Monistrol y otros, 2006) y el reciente del 2009. Los datos se muestran en valores absolutos. Destaca especialmente en positivo el Museo de Historia de Cataluña entre los grandes con un aumento superior a los treinta y nueve mil enlaces. Entre los locales destaca el Museo de Granollers que gana en estos tres años más de catorce mil enlaces. En negativo la caída en más de ocho mil de la Fundación Miró.

\section{TABLA II}

Comparativa de estudios de visibilidad 2006-2009*

\begin{tabular}{|c|c|c|c|c|}
\hline Museo (ordenado por diferencia) & 2006 & 2009 & $\begin{array}{c}\text { Diferencia } \\
2006-2009\end{array}$ & $\begin{array}{c}\% \\
\text { Diferencia } \\
2006-2009\end{array}$ \\
\hline Museu d'Història de Catalunya & 388 & 39.618 & 39.230 & 10.111 \\
\hline Museu de Granollers & 189 & 14.486 & 14.297 & 7.565 \\
\hline Fundació Dalí & 5.150 & 16.972 & 11.822 & 230 \\
\hline Museu Nacional d'Art de Catalunya & 1.600 & 10.948 & 9.348 & 584 \\
\hline Museu Picasso & 1.920 & 9.700 & 7.780 & 405 \\
\hline Museu d'Art Contemporani de Barcelona & 655 & 6.274 & 5.619 & 858 \\
\hline Museu Diocesà de Tarragona & 201 & 2.069 & 1.868 & 929 \\
\hline Museu d'Història de Barcelona & 101 & 1.912 & 1.811 & 1.793 \\
\hline Museu Comarcal de l'Urgell & 876 & 2.276 & 1.400 & 160 \\
\hline Museu de la Música & 39 & 1.404 & 1.365 & 3.500 \\
\hline Museu de Zoologia & 12 & 7 & -5 & -42 \\
\hline Museu d'Història de Cambrils & 57 & 34 & -23 & -40 \\
\hline Museu Diocesà d'Urgell & 84 & 53 & -31 & -37 \\
\hline Catedral de Girona & 70 & 1 & -69 & -99 \\
\hline Museu del Joguet de Catalunya & 595 & 513 & -82 & -14 \\
\hline Museu del Futbol Club Barcelona & 174 & 8 & -166 & -95 \\
\hline Museu de la Pell d'Igualada & 170 & 1 & -169 & -99 \\
\hline Museu-Arxiu Municipal de Calella & 193 & 13 & -180 & -93 \\
\hline Biblioteca Museu Víctor Balaguer & 2.190 & 178 & -2.012 & -92 \\
\hline Fundació Miró & 10.500 & 2.229 & -8.271 & -79 \\
\hline
\end{tabular}

* Los 10 mejores y los 10 peores en diferencia. Ver tabla completa en el anexo.

Las medias globales de los análisis realizados, nos muestra un aumento muy importante en el período 2006-2009. En estos tres años los museos catalanes han aumentado de promedio el número de enlaces en 1.465 , lo que supone un aumento del 326\%. 
Hemos constatado que 39 de los 61 museos analizados (65\%) han aumentado significativamente el número de enlaces recibidos y que tan solo 12 (19\%) han disminuido. Por otro lado, es necesario resaltar lo siguiente:

- 2 museos han aumentado por encima del 10.000\%

- 9 museos han aumentado por encima del 1.000\%

- 15 museos han aumentado por encima del 500\%

Como síntesis podemos afirmar que el aumento global en el número de enlaces recibidos entre el 2006 y el 2009 de los sitios web de los museos de Cataluña ha sido posible gracias a los grandes aumentos del $25 \%$ de los museos estudiados, en especial de los cuatro primeros que acumulan el $62 \%$ del total del aumento global entre 2006 y 2009 (tabla III).

\section{TABLA III}

Síntesis comparativa 2006-2009 de los mejores resultados de los museos

\begin{tabular}{l|r|r|r|r|r}
\hline \multicolumn{1}{c|}{$\begin{array}{c}\text { Museos (ordenados por porcentaje } \\
\text { de aumento) }\end{array}$} & $\mathbf{2 0 0 6}$ & $\mathbf{2 0 0 9}$ & Diferencia & $\begin{array}{c}\text { Diferencia } \\
\text { acumulada }\end{array}$ & $\begin{array}{c}\text { \% } \\
\text { aumento } \\
\text { acumulado }\end{array}$ \\
\hline $\begin{array}{l}\text { Museu Nacional de la Ciència i la Tèc- } \\
\text { nica de Catalunya }\end{array}$ & 9 & 1.148 & 1.139 & 1.139 & 1,27 \\
\hline Museu d'Història de Catalunya & 388 & 39.618 & 39.230 & 40.369 & 45,16 \\
\hline Museu Municipal Josep Aragay (Breda) & 6 & 526 & 520 & 40.889 & 45,47 \\
\hline Museu de Granollers & 189 & 14.486 & 14.297 & 55.186 & 61,73 \\
\hline Total de los 4 mejores & 592 & 55.778 & 55.186 & & \\
\hline Total global de los 61 estudiados & 27.384 & 116.678 & 89.394 & & \\
\hline
\end{tabular}

A pesar de que el análisis del Page Rank queda fuera de los objetivos de este estudio, hemos comprobado la relación entre el número de enlaces recibidos (escala lineal) y el Page Rank (escala logarítmica) que Google otorga a webs. Esta comprobación también se ha realizado teniendo en cuenta el aumento o la disminución de los enlaces recibidos.

Como podemos ver (tabla IV), casi todos los museos del grupo que han ganado más visibilidad también han aumentado su Page Rank. Tan solo tres museos mantienen el mismo Page Rank y ninguno de ellos disminuye. Por otra parte, entre los museos que pierden más visibilidad, tres mantienen el indicador, uno, curiosamente, lo aumenta y los seis restantes pierden significativamente puntuación.

A pesar de que la muestra no es suficientemente significativa, los resultados confirman una idea básica del posicionamiento web: el Page Rank depende en buena parte, pero no exclusivamente, del número de enlaces entrantes. 
TABLA IV

PageRank de los museos con mayor aumento y pérdida de visibilidad

\begin{tabular}{l|c|c}
\hline \multicolumn{1}{c|}{ Museos que han ganado visibilidad y Page Frank } & $\mathbf{2 0 0 6}$ & $\mathbf{2 0 0 9}$ \\
\hline Museu d'Història de Catalunya & 5 & 6 \\
\hline Museu de Granollers & 4 & 5 \\
\hline Fundació Dalí & 6 & 6 \\
\hline Museu Nacional d'Art de Catalunya & 6 & 6 \\
\hline Museu Picasso & 6 & 6 \\
\hline Museu d'Art Contemporani de Barcelona & 4 & 7 \\
\hline Museu Diocesà de Tarragona & 4 & 5 \\
\hline Museu d'Història de Barcelona & 4 & 5 \\
\hline Museu Comarcal de l'Urgell & 4 & 5 \\
\hline Museu de la Música & $\mathbf{2 0 0 6}$ & $\mathbf{2 0 0 9}$ \\
\hline \multicolumn{1}{c|}{ Museos que han perdido visibilidad y PageRank } & 4 & 1 \\
\hline Museu de Zoologia & 3 & 3 \\
\hline Museu d'Història de Cambrils & 3 & 0 \\
\hline Museu Diocesà d'Urgell & 2 & 0 \\
\hline Catedral de Girona & 5 & 5 \\
\hline Museu del Joguet de Catalunya & 4 & 4 \\
\hline Museu del Futbol Club Barcelona & 4 & 0 \\
\hline Museu de la Pell d'Igualada & 4 & 2 \\
\hline Museu-Arxiu Municipal de Calella & 6 & 5 \\
\hline Biblioteca Museu Víctor Balaguer & & 5 \\
\hline Fundació Miró & 4 & 5 \\
\hline
\end{tabular}

En la comparación de la visibilidad de los principales museos catalanes con los del grupo de control Europa y con los de dominio .museum, podemos constatar lo siguiente (tabla V):

- La web del mejor museo de Cataluña en visibilidad ocuparía la cuarta posición entre los mejores de Europa y la segunda entre los mejores del dominio .museum.

- El promedio de enlaces recibidos de los 10 mejores museos en visibilidad de Cataluña es comparable al promedio de los 10 mejores del dominio .museum.

- Las sedes web de los tres mejores museos en visibilidad de Europa tienen entre 2 y 3 veces más enlaces recibidos que el mejor de Cataluña.

Las mejores sedes web de los museos de Cataluña tienen un buen nivel internacional en visibilidad aunque están todavía lejos de los mejores de Europa. 


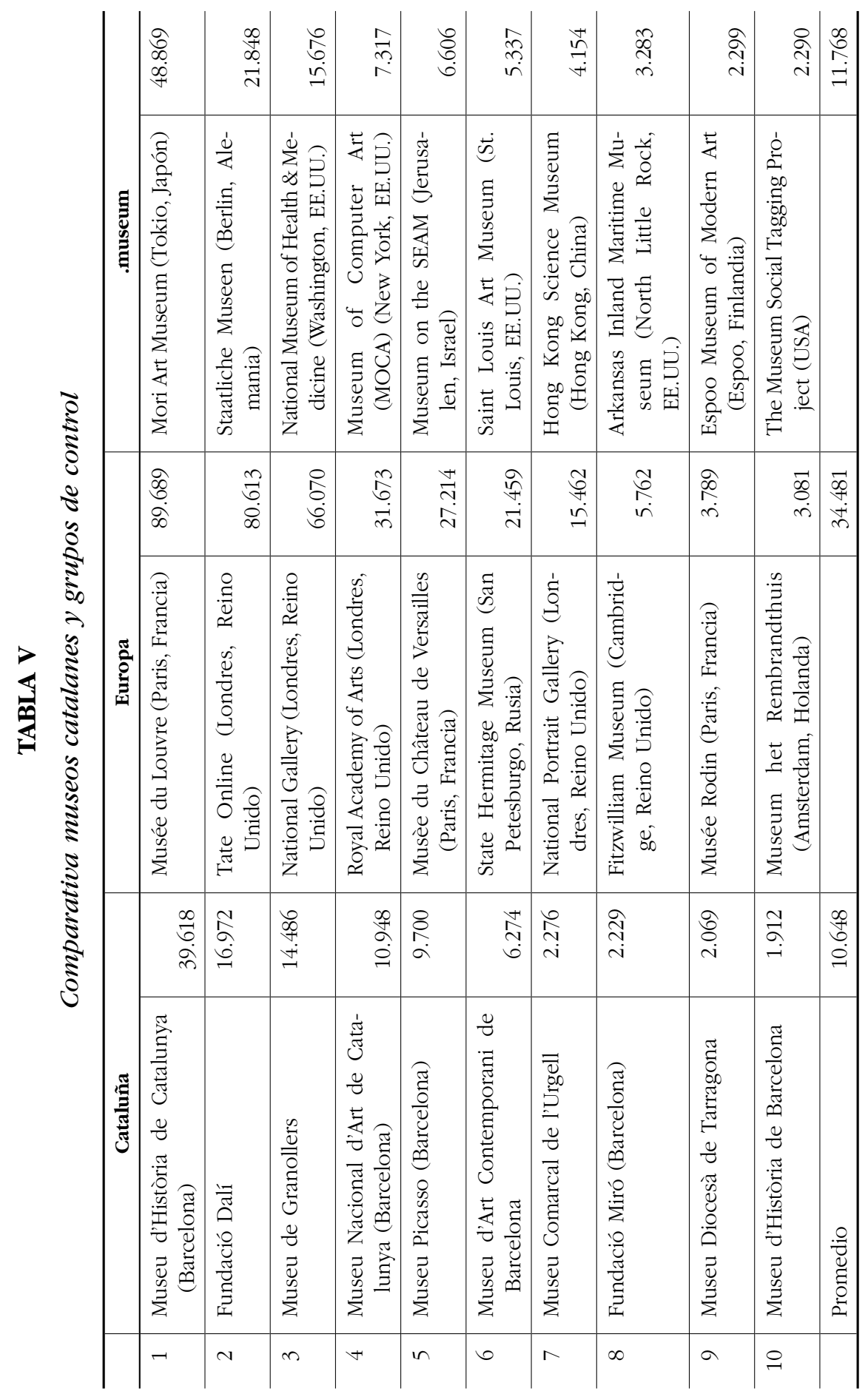




\section{Análisis de los resultados}

Resulta un tanto complejo afirmar si un determinado aumento de enlaces entrantes es o no significativo. Todavía es más comprometido sacar conclusiones sobre la evolución positiva de la presencia de los museos catalanes en Internet en base a estos datos. No obstante, el aumento parece significativo si lo comparamos con los valores de aumento de los enlaces recibidos de algunas sedes web de referencia.

\section{TABLA VI}

Visibilidad de sedes web de referencia. Comparativa datos 2006 y 2009

\begin{tabular}{l|r|r|r|r}
\hline \multicolumn{1}{c|}{ Sede web } & \multicolumn{1}{c|}{$\mathbf{2 0 0 6}$} & \multicolumn{1}{c|}{$\mathbf{2 0 0 9}$} & \multicolumn{1}{c}{$\begin{array}{c}\text { Diferencia } \\
\mathbf{2 0 0 6 - 2 0 0 9}\end{array}$} & $\begin{array}{c}\text { \% } \\
\text { Diferencia } \\
\mathbf{2 0 0 6 - 2 0 0 9}\end{array}$ \\
\hline Ayuntamiento de Barcelona & 480.703 & 153.198 & -327.505 & $-213,78$ \\
\hline Directorio DMOZ & 813.000 & 3.172 .465 & 2.359 .465 & 74,37 \\
\hline Congreso de Diputados de España & 49.073 & 40.465 & -8.608 & $-21,27$ \\
\hline Google & 71.500 .000 & 257.191 .449 & 185.691 .449 & 72,20 \\
\hline WebLog OJO!Buscador & 20.700 & 67.076 & 46.376 & 69,14 \\
\hline TV3 & 760 & 4.775 & 4.015 & 84,08 \\
\hline
\end{tabular}

Por razones obvias, la selección de sedes web de referencia no pretende ser una muestra representativa desde un punto de vista estadístico. Es tan solo un grupo de sedes web significativas de sectores diversos que permiten tener un punto de referencia para valorar los resultados obtenidos en las sedes web de los museos de Cataluña. Como podemos ver, los aumentos del número de enlaces recibidos por las webs de referencia no superan en ningún caso el 85\%. Por tanto, teniendo en cuenta estos referentes, podemos afirmar que el aumento de un $326 \%$ experimentado por las sedes web de museos de Cataluña es muy importante.

Por otra parte, no es descartable que los valores sobre el número de resultados que ofrecen los buscadores contengan errores. Son estimaciones aproximadas de los resultados reales y no cálculos precisos de un conjunto de registros de una base de datos. Los buscadores potencian la rápida respuesta a costa de no recuperar físicamente todos los resultados pertinentes y por tanto se ven en la obligación de aplicar un algoritmo de estimación del número total de resultados.

Hay una larga bibliografía sobre la fiabilidad de los resultados de los buscadores (Rousseau, 1998 y 1999; Notess, 2000; Thelwall, 2001; Mettrop, 2001; BarIlan, 2005; Lewandowski, 2008; Uyar, 2009). Investigaciones recientes apuntan que Yahoo es más fiable que Google en las estimaciones del número de resul- 
tados que ofrece en sus búsquedas. No obstante no sabemos con qué margen de error nos estamos moviendo, tampoco si se han producido cambios en los dos últimos años en los algoritmos de estimación que puedan haber afectado a esta investigación.

Pensamos que el escenario más plausible es aquél en el que el algoritmo de estimación no se ha modificado de forma significativa y, por tanto, el margen de error de la recogida de datos del año 2006 es equiparable al posible error del 2009. En este caso, los datos serían comparables y el aumento de enlaces recibidos detectado correspondería a datos reales, al menos en valores porcentuales.

\section{Conclusiones}

Los museos catalanes aumentan visibilidad en el período 2006-2009 en un $326 \%$. A pesar de la excelencia de la cifra, hay que tener en cuenta que el aumento está concentrado en 15 (25\%) museos que han aumentado el número de enlaces recibidos por encima del $500 \%$. En general los museos pequeños han aumentado muy poco su visibilidad.

Sospechamos que una de las principales razones de este más que significativo aumento en este grupo de 15 museos, es debido a acciones específicas y explícitas y no como consecuencia de un crecimiento espontáneo. Acciones como por ejemplo campañas encaminadas a la mejora del posicionamiento. Esta sospecha deberá confirmarse en posteriores estudios.

Por otra parte, todavía no tenemos una explicación global de los motivos de la pérdida significativa de visibilidad de un grupo de 10 ó 12 sedes web. En cambio, sí que hemos podido constatar que un cambio en la URL ha sido la causa de la pérdida de más de 8.000 enlaces en la Fundación Miró. Este cambio ha tenido consecuencias extremadamente negativas en la cantidad de enlaces recibidos, como no podría ser de otra manera.

El estudio comparativo con los grupos de control internacionales nos indica que las mejores sedes web de los museos de Cataluña tienen un nivel internacional en visibilidad aunque están lejos todavía de los mejores de Europa.

Quedan abiertas nuevas líneas de investigación, estudio y debate, en especial para identificar si hay diferencias significativas en visibilidad en función del tipo, el tamaño o el presupuesto de la institución museística o el tamaño, la usabilidad o los contenidos de las sedes web de los museos. Estos nuevos estudios deben profundizar en la identificación de buenas prácticas para la mejora de la visibilidad y en la valoración del impacto sociocultural de las sedes web de los museos.

\section{Bibliografía}

Aguillo, I. F. (2000). Proposal for a research agenda for cybermetric studies. Proceedings $7^{\text {th }}$ International Conference of the International Society for Scientometrics and Informetrics. Colima, México.

Rev. Esp. Doc. Cient., 33, 4, octubre-diciembre, 531-552, 2010. ISSN: 0210-0614. doi:10.3989/redc.2010.4.754 543 
Aguillo, I. F. (1998). STM information on the web and the development of new Internet R\&D databases and indicators. Proceedings Online Information Meeting. 239-243. London.

Aguillo, I. F. (2002). Cybermetrics, definitions and methods for an emerging discipline. Séminaires de l'ADEST, Paris, 14 February 2002. < http://www.upmf-grenoble.fr/adest/ seminaires/ISIDRO/Cybermetrics.ppt> [consulta: 25 de septiembre de 2009].

Almind, T. C., y Ingwersen, P. (1997). Informetric analyses on the World Wide Web: a methodological approach to "Webometrics". Journal of Documentation, vol. 53 (4), 404-426.

Alonso Berrocal, J. L.; Figuerola, C. G., y Zazo, A. F. (2004). Cibermetría: nuevas técnicas de estudio aplicables al web. Gijón; Trea., 124-125.

Baeza-Yates, R., y Davis, E. (2004). Web page ranking using link attributes. Proceedings International Web Wide World Conference. 328-329. New York.

Baeza-Yates, R.; Boldi, P., y Castillo, C. (2006). Generalizing PageRank: damping functions for link-based ranking algorithms. Proceedings Annual ACM Conference on Research and Development in Information Retrieval, 308-315. Seattle.

Bar-Ilan, J. (2005). Expectations versus reality - Search engine features needed for web search at mid. International Journal of Scientometrics, Informetrics and Bibliometrics, vol. 9 (1). http://www.cindoc.csic.es/cybermetrics/articles/v9ip2.htm [consulta: 25 de septiembre de 2009].

Bray, T. (1996). Measuring the web. Computer networks and ISDN systems, vol. 28 (7-11), 993-1005.

Burnette, A. (2009). Redesigning your museum's web site: a survivors' guide. En: Trant, J.; Bearman, D. (editores). Museums and the Web 2009: proceedings. Archives and Museum Informatics, Toronto, Canadá.

Codina, L. (2000). Evaluación de recursos digitales en línea: conceptos, indicadores y métodos. Revista Española de Documentación Científica, v. 23 (1), 9-44.

Corporación Internacional de Fomento del Negocio Electrónico (CIFNE S.L.) Extracto del Informe corporativo de museos estatales (2001): http://www.acctiva.com/recursos/ AcctivaMuseos.pdf [consulta: 25 de septiembre de 2009].

Cunliffe, D.; Kritou, E., y Tudhope, D. Usability evaluation for museum web sites. Museum Management and Curatorship, vol. 19 (3), 229-252.

Directorio de los Museos de España. [on line] Madrid: Ministerio de Cultura. http://www. mcu.es/directoriomuseo/loadSearch.do?cache=init\&layout=directoriomuseo\&language =es [consulta: 25 de septiembre de 2009].

Dyson, M. C., y Moran, K. (2000). Informing the design of web interfaces to museum collections. Museum Management and Curatorship, vol. 18 (4), 391-406.

Garzotto, F.; Matera, M., y Paolini, P. (1998). To use or not to use? Evaluating usability of museum web sites. Proceedings Museums and the Web: an International Conference. Toronto, Canadá.

Harms, I., y Werner, S. (2001). Evaluating the usability of a museum web site. Museums and the Web 2001: proceedings. $5^{\text {th }}$ International Conference. Seattle.

Henning, M. (2006). Museums, media and cultural theory. New York: Open University Press.

Hertzum, M. (1999). A review of museum web sites: in search of user-centred design. Archives and Museum Informatics, vol. 12 (2). 
Ingwersen, P. (1998). The calculation of web impact factors. Journal of Documentation, vol. 54 (2), 236-243.

Kravchyna, V., y Hastings, S. (2002). Informational value of museum web sites. First Monday, vol. 7 (2)

Lewandowski, D. (2008). Three-year on the freshness of web search engine database. Journal of Information Science, vol. 34 (6), 817-831. http://jis.sagepub.com/content/34/6/817.abstract [consulta: 25 de septiembre de 2009].

López de Prado, R. (1998). Museos en internet: análisis de recursos documentales. Fesabid 98: IV Jornadas Españolas de Información y Documentación.

Mettrop, W. (2001). Internet search engines: fluctuations in document accessibility. Journal of Documentation, vol. 57 (5).

Monistrol, R. (2005). Difusió cultural i webs de museus on line: estat de la qüestió. Trabajo de investigación. Doctorado bienio 2001-2003 en Comunicación Social, Universitat Pompeu Fabra.

Monistrol, R.; Rovira, C., y Codina, L. (2006). Sitios Web de museos de Cataluña: análisis y propuesta de evaluación [on line]. Hipertext.net, núm. 4 [consulta: 10 de enero de 2009].

Mulholland, P.; Collins, T.; Zdrahal, Z. (2005). Bletchley park text: using mobile and semantic web technologies to support the post-visit use of online museum resources. Journal of Interactive Media in Education (24), 1-21.

Notess, G. (2000). Search Engine Inconsistencies. Online, March.

Noruzi, A. (2006). The web impact factor: a critical review. The Electronic Library, vol. 24 (4), 490-500.

Olsina, L. A. (1999). Metodología cuantitativa para la evaluación y comparación de la calidad de sitios web. Tesis doctoral. Facultad de Ciencias Exactas, Universidad de la Plata (Argentina).

Pallas, J. (2008). Economides, A. A. Evaluation of art museums' web sites worldwide. IOS Press, vol. 28 (1).

Pardo Kuklinski, H. (2008). Sitios web institucionales de museos: un modelo de análisis hacia la web 2.0. En: Mateos Rusillo, S. M. (coord.). La comunicación global del patrimonio cultural. Gijón; Trea, 380-396.

Patrimoni Gencat. Museus [on line]. Barcelona: Generalitat de Cataluña http://cultura.gencat.net/museus/muscerca1.asp [consulta: 28 de septiembre de 2009].

Pedraza, R.; Codina, L.; Rovira, C. (2008). Semantic Web adoption: online tools for web evaluation and metadata extraction. En: Da Ruan y otros (eds.) Computational Intelligence In Decision And Control. Proceedings $8^{\text {th }}$ International Flins Conference, 2008, World Scientific Publishing Co., New Jersey.

Rodríguez i Gairín, J.M. Valorando el impacto de la información en internet. http://www. kronosdoc.com/jm.htm [consulta: 10 de enero de 2009].

Rousseau, R. (1998). Sitations: an exploratory study. Cybermetrics, vol. 1 (1). http://www. cindoc.csic.es/cybermetrics/articles/v1i1p1.html [consulta: 25 de septiembre de 2009].

Rousseau, R. (1998). Daily time series of common single word searches in Altavista and Northerm Ligh. International Journal of Scientometrics, Informetrics and Bibliometrics, vol. $2 / 3(1)$.

Rev. Esp. Doc. Cient., 33, 4, octubre-diciembre, 531-552, 2010. ISSN: 0210-0614. doi:10.3989/redc.2010.4.754 545 
Rovira, C. (2007). Artículo M529. Rastreo automático: DigiDocSpider [en linea]. En: Rovira, C.; Codina, L. (directores.). Documentación digital. Barcelona: Área de Ciencias de la Documentación. Departamento de Periodismo y de Comunicación Audiovisual. Universidad Pompeu Fabra, http://www.documentaciondigital.org [consulta: 3 de noviembre de 2008].

Rovira, C. (2008). Visibilitat dels llocs webs culturals de Catalunya. I Jornada Difusió Cultural $i$ World Wide Web. Barcelona, España: Facultat de Biblioteconomia i Documentació, Universitat de Barcelona. http://eprints.rclis.org/16352/1/jornades_ub_2008.pdf.

Sharples, M.; Lonsdale, P.; Meek, J.; Rudman, P. D., y Vavoula, G. N. (2007). An evaluation of my art space: a mobile learning service for school museum trips. Proceedings of Learn, Melbourne, Australia.

Snyder, H., y Rosenbaum, H. (1999). Can search engines be used as tools for web-link analysis? A critical view. Journal of documentation, vol. 55 (4), 375-384.

Thelwall, M. A. (2004). Link analysis: an information science approach. Amsterdam: Elservier.

Thelwall, M. A. (2001). The responsiveness of search engines. International Journal of Scientometrics, Informetrics and Bibliometrics, vol. 5 (1).

Uyar, A. (2009). Investigation of accuracy of search engine hit counts. Journal of Information Science, vol. 35 (4), 469-480.

Nota: este artículo ha sido elaborado en el contexto del proyecto de investigación CSO2008-02627 "Nuevas estrategias de publicidad y promoción de marcas turísticas españolas en la web" y del trabajo de fin de estudios del Master en Gestión de Contenidos Digitales (2008/2009).

\section{Anexo 1. Sedes Web de museos de Cataluña}

\begin{tabular}{l|l}
\hline \multicolumn{1}{c|}{ Museo (ordenación alfabética) } & \multicolumn{1}{c}{ Dirección web } \\
\hline $\begin{array}{l}\text { Biblioteca Museu Víctor Balaguer } \\
\text { (Vilanova i la Geltrú) }\end{array}$ & http://www.victorbalaguer.cat/ \\
\hline Catedral de Girona & $\begin{array}{l}\text { http://www.catedraldegirona.org/visita/cat/tresor/principal/ } \\
\text { index.html }\end{array}$ \\
\hline Centre d'Interpretació del Parc Güell & http://www.bcn.cat/museuhistoriaciutat/ca/park_guell.html \\
\hline Fundació Dalí & http://www.salvador-dali.org/index.html \\
\hline Fundació Miró & http://fundaciomiro-bcn.org/ \\
\hline Fundació Tàpies & http://www.fundaciotapies.org/site/spip.php?rubrique65 \\
\hline $\begin{array}{l}\text { L'Enrajolada Casa - Museu Santacana } \\
\text { (Martorell) }\end{array}$ & $\begin{array}{c}\text { http://www.martorell.cat/ajmartorell/ca/arees-municipals/ } \\
\text { cultura/museus/enrajolada.html }\end{array}$ \\
\hline $\begin{array}{l}\text { Museu Agrícola de Cambrils } \\
\text { Museu Comarcal de l'Urgell }\end{array}$ & http://www.cambrils.org/mhc/ct/agricola.htm \\
\hline
\end{tabular}

546 Rev. Esp. Doc. Cient., 33, 4, octubre-diciembre, 531-552, 2010. ISSN: 0210-0614. doi:10.3989/redc.2010.4.754 


\begin{tabular}{|c|c|}
\hline Museo (ordenación alfabética) & Dirección web \\
\hline Museu d'Arqueologia de Catalunya & http://www.mac.cat/ \\
\hline $\begin{array}{l}\text { Museu d'Art Contemporani de Bar- } \\
\text { celona }\end{array}$ & http://www.macba.es/controller.php \\
\hline Museu d'Art de Girona & http://www.museuart.com/cat/index.html \\
\hline Museu d'Art Jaume Morera (Lleida) & http://www.paeria.es/mmorera/ \\
\hline Museu d'Art Modern de Tarragona & http://www.altanet.org/MAMT/CAT/mamt.htm \\
\hline Museu d'Història de Barcelona & $\begin{array}{l}\text { http://w3.bcn.es/V64/Home/V64XMLHomeLinkPl/ } \\
\text { 0,4468,335907851_335943991_1,00.html }\end{array}$ \\
\hline Museu d'Història de Cambrils & http://www.cambrils.org/mhc/ct/index.htm \\
\hline Museu d'Història de Catalunya & http://www.mhcat.net/ \\
\hline Museu d'Història de Girona & http://www.ajuntament.gi/museu_ciutat/ \\
\hline Museu d'Història de Sabadell & http://www.ajsabadell.net \\
\hline Museu de Badalona & http://www.museubdn.es/ \\
\hline Museu de Cardedeu & http://www.museudecardedeu.org/ \\
\hline Museu de Cervera & http://www.museudecervera.cat/start.htm \\
\hline Museu de Gavà & http://www.patrimonigava.com/cat/imgpcn/mg.asp \\
\hline Museu de Granollers & http://www.museugranollers.org/ \\
\hline Museu de l'Empordà & http://www.museuemporda.org/ \\
\hline $\begin{array}{l}\text { Museu de l'Institut Botànic de Bar- } \\
\text { celona }\end{array}$ & http://www.institutbotanic.bcn.es/ \\
\hline Museu de la Música & $\begin{array}{l}\text { http://w3.bcn.es/V62/Home/V62XMLHomeLinkPl/ } \\
\text { 0,4388,285254511_285742177_1,00.html }\end{array}$ \\
\hline Museu de la Noguera & http://www.museucn.com/catala/index.htm \\
\hline Museu de la Pell d'Igualada & http://www.igualada.cat/web/seccio2.php?Id=410 \\
\hline Museu de la Pesca de Palamós & http://www.museudelapesca.org/web/ \\
\hline Museu de Montserrat & http://www.museudemontserrat.com/ \\
\hline Museu de Ripio & http://www.museuderipoll.org/ \\
\hline Museu de Terrassa & $\begin{array}{l}\text { http://www.ajterrassa.es/ajuntament/imcet/cultura/museus/ } \\
\text { museudeterrassa/index.html }\end{array}$ \\
\hline Museu de Zoología & http://www.bcn.es/medciencies/catala/museozoo.htm \\
\hline Museu del Cinema (Girona) & http://www.museudelcinema.org/ct/c1.html \\
\hline Museu del Futbol Club Barcelona & $\begin{array}{l}\text { http://www.fcbarcelona.com/web/catala/club/club_avui/te- } \\
\text { rritori_barca/museu/museu.html }\end{array}$ \\
\hline Museu del Joguet de Catalunya & http://www.mjc.cat/ \\
\hline Museu del Suro de Palafrugell & http://www.museudelsuro.cat/ \\
\hline Museu del Vidre de Vimbodí & http://www.vimbodi.altanet.org/museudelvidre.php \\
\hline
\end{tabular}




\begin{tabular}{|c|c|}
\hline Museo (ordenación alfabética) & Dirección web \\
\hline Museu Deu (Vendrell) & $\begin{array}{l}\text { http://www.elvendrell.net/PaginaM1IndexLit.aspx?MWS } \\
\text { IdElem=101014\&MWS_Tip=Obj\&MWS_IDRCate= } \\
\text { VIMusDe }\end{array}$ \\
\hline Museu Diocesà d'Urgell & http://www.museudiocesaurgell.org/home/cat/benvingut.htm \\
\hline Museu Diocesà de Tarragona & http://museu.diocesa.arquebisbattarragona.cat/ \\
\hline Museu Episcopal de Vic & http://www.museuepiscopalvic.com/ \\
\hline Museu Etnològic del Montseny & http://www.museuetnologicmontseny.org/ \\
\hline Museu Frederic Marès & http://www.museumares.bcn.es/index.htm \\
\hline $\begin{array}{l}\text { Museu Geològic del Seminari de Bar- } \\
\text { celona }\end{array}$ & http://www.bcn.es/medciencies/mgsb/ \\
\hline Museu Marítim de Barcelona & $\begin{array}{l}\text { http: } / / \text { www.mmb.cat } / \text { default } \text { asp?idApartado=96\&id } \\
\text { Idioma }=1\end{array}$ \\
\hline Museu Molí Paperer de Capellades & http://www.mmp-capellades.net/catala/default.htm \\
\hline Museu Monestir de Pedralbes & $\begin{array}{l}\text { http://w3.bcn.es/V64/Home/V64XMLHomeLinkPl/ } \\
\text { 0,4468,335907851_335943991_1,00.html }\end{array}$ \\
\hline $\begin{array}{l}\text { Museu Municipal de Nàutica del } \\
\text { Masnou }\end{array}$ & http://www.elmasnou.net/ambit.php?id=6 \\
\hline Museu Municipal de Tossa de Mar & http://www.tossademar.com/museu/home.htm \\
\hline $\begin{array}{l}\text { Museu Municipal Josep Aragay } \\
\text { (Breda) }\end{array}$ & http://www.museuaragay.org/ \\
\hline $\begin{array}{l}\text { Museu Municipal Vicenç Ros (Mar- } \\
\text { torell) }\end{array}$ & $\begin{array}{l}\text { http://www.martorell.cat/ajmartorell/ca/arees-municipals/ } \\
\text { cultura/museus/vincenc-ros.html }\end{array}$ \\
\hline Museu Nacional d'Art de Catalunya & http://www.mnac.cat/index.jsp?lan=001 \\
\hline $\begin{array}{l}\text { Museu Nacional de la Ciència i la } \\
\text { Tècnica de Catalunya }\end{array}$ & http://www.mnactec.cat/ \\
\hline Museu Pau Casals & http://www.paucasals.org/ca/MUSEU-historia-i-missio/ \\
\hline Museu Picasso & $\begin{array}{l}\text { http://w3.bcn.es/V66/Home/V66XMLHomeLinkPl/ } \\
\text { 0,4589,417470534_417617303,00.html }\end{array}$ \\
\hline Museu Salvador Vilaseca (Reus) & http://museus.reus.net/msv/ \\
\hline Museu Tèxtil i d'Indumentària & $\begin{array}{l}\text { http://www.dhub-bcn.cat/ca/museus/museu-textil-i-dindu- } \\
\text { mentaria }\end{array}$ \\
\hline Museu Torre Balldovina & http://www.minorisa.es/mtbsc/ \\
\hline Museu-Arxiu Municipal de Calella & $\begin{array}{l}\text { http://www.calella.cat/atencio_al_ciutada/equipaments/Cul- } \\
\text { turals/Museu_Arxiu_Municipal_de_Calella_Josep_M._Co- } \\
\text { dina_i_Bague_ }\end{array}$ \\
\hline
\end{tabular}




\section{Anexo 2. Sedes web de museos de Europa}

\begin{tabular}{|c|c|}
\hline Museo (ordenación alfabética) & Dirección web \\
\hline $\begin{array}{l}\text { Art and Exhibition Hall of the Federal Re- } \\
\text { public of Germany }\end{array}$ & http://www.kah-bonn.de/index_e.htm \\
\hline Fitzwilliam Museum & http://www.fitzmuseum.cam.ac.uk/ \\
\hline Kunsthistorisches Museum Vienna & http://www.khm.at/khm-portal/ \\
\hline Musèe du Château de Versailles & http://www.chateauversailles.fr/homepage \\
\hline Musée du Louvre & http://www.louvre.fr/llv/commun/home.jsp \\
\hline Musée Rodin & http://www.musee-rodin.fr/accueil.htm \\
\hline Museum het Rembrandthuis & $\begin{array}{l}\text { http://www.rembrandthuis.nl/cms_pages/index } \\
\text { main.html }\end{array}$ \\
\hline National Gallery & http://www.nationalgallery.org.uk/ \\
\hline National Museum of Denmark & http://www.nationalmuseet.dk/sw20374.asp \\
\hline National Portrait Gallery & http://www.npg.org.uk/index.php \\
\hline Rijksmuseum Amsterdam & http://www.rijksmuseum.nl/index.jsp?lang=en \\
\hline Royal Academy of Arts & http://www.royalacademy.org.uk/ \\
\hline State Hermitage Museum & $\begin{array}{l}\text { http://www.hermitagemuseum.org/html_En/index. } \\
\text { html }\end{array}$ \\
\hline Tate Online & http://www.tate.org.uk/ \\
\hline The State Russian Museum & http://www.rusmuseum.ru/eng/home/ \\
\hline
\end{tabular}

\section{Anexo 3. Museos con dominio .museum}

\begin{tabular}{|c|c|}
\hline Museo & Dirección web \\
\hline Mori Art Museum (Tokio, Japón) & http://www.mori.art.museum \\
\hline Staatliche Museen (Berlin, Alemania) & http://www.smb.museum \\
\hline $\begin{array}{l}\text { National Museum of Health \& Medicine } \\
\text { (Washington, EE.UU.) }\end{array}$ & http://nmhm.washingtondc.museum \\
\hline $\begin{array}{l}\text { Museum of Computer Art (MOCA) (New } \\
\text { York, EE.UU.) }\end{array}$ & http://moca.virtual.museum \\
\hline Museum on the SEAM (Jerusalen, Israel) & http://www.coexistence.art.museum \\
\hline Saint Louis Art Museum (St. Louis, EE.UU.) & http://www.stlouis.art.museum \\
\hline $\begin{array}{l}\text { Hong Kong Science Museum (Hong Kong, } \\
\text { China) }\end{array}$ & http://hk.science.museum \\
\hline $\begin{array}{l}\text { Arkansas Inland Maritime Museum (North } \\
\text { Little Rock, EE.UU.) }\end{array}$ & http://aimm.museum \\
\hline $\begin{array}{l}\text { Espoo Museum of Modern Art (Espoo, Fin- } \\
\text { landia) }\end{array}$ & http://www.emma.museum \\
\hline
\end{tabular}




\begin{tabular}{|c|c|}
\hline Museo & Dirección web \\
\hline The Museum Social Tagging Project (EE.UU.) & http://www.steve.museum \\
\hline $\begin{array}{l}\text { Museum of Archaeology \& Etnology (Bur- } \\
\text { naby, Canadá) }\end{array}$ & http://www.sfu.museum \\
\hline $\begin{array}{l}\text { Virginia Museum of Fine Arts (Richmond, } \\
\text { EE.UU.) }\end{array}$ & http://www.vmfa.museum \\
\hline Nobel Foundation (Stockholm, Suecia) & http://nobelprize.virtual.museum \\
\hline $\begin{array}{l}\text { RAF Manston Spitfire \& Hurricane Memorial } \\
\text { Museum (Ramsgate, Inglaterra) }\end{array}$ & http://spitfire.memorial.museum \\
\hline $\begin{array}{l}\text { National children's Museum (National Har- } \\
\text { bor, EE.UU.) }\end{array}$ & http://www.ncm.museum \\
\hline $\begin{array}{l}\text { Southern Appalachia Railway Museum (Oak } \\
\text { Ridge, EE.UU.) }\end{array}$ & http://www.southernappalachia.railway.museum \\
\hline The British Museum (Londres, Inglaterra) & http://the.british.museum \\
\hline $\begin{array}{l}\text { The Library amd Museum of Freemasonry } \\
\text { (Londres, Inglaterra) }\end{array}$ & http://www.freemasonry.london.museum \\
\hline Silk Museum (Macclesfield, Inglaterra) & http://www.macclesfield.silk.museum \\
\hline Dr. Sun Yat-sen Museum (Hong Kong, China) & http://hk.drsunyatsen.museum \\
\hline
\end{tabular}

\section{Anexo 4. Clasificación de visibilidad (2006-2009) valores absolutos}

\begin{tabular}{l|c|c|c|c}
\hline \multicolumn{1}{c|}{ Museo (ordenado por diferencia) } & $\mathbf{2 0 0 6}$ & $\mathbf{2 0 0 9}$ & $\begin{array}{c}\text { Diferencia } \\
\mathbf{2 0 0 6 - 2 0 0 9}\end{array}$ & $\begin{array}{c}\text { \% } \\
\text { Diferencia } \\
\mathbf{2 0 0 6}-2009\end{array}$ \\
\hline Museu d'Història de Catalunya & 388 & 39.618 & 39.230 & 10.111 \\
\hline Museu de Granollers & 189 & 14.486 & 14.297 & 7.565 \\
\hline Fundació Dalí & 5.150 & 16.972 & 11.822 & 230 \\
\hline Museu Nacional d'Art de Catalunya & 1.600 & 10.948 & 9.348 & 584 \\
\hline Museu Picasso & 1.920 & 9.700 & 7.780 & 405 \\
\hline Museu d'Art Contemporani de Barcelona & 655 & 6.274 & 5.619 & 858 \\
\hline Museu Diocesà de Tarragona & 201 & 2.069 & 1.868 & 929 \\
\hline Museu d'Història de Barcelona & 101 & 1.912 & 1.811 & 1793 \\
\hline Museu Comarcal de l'Urgell & 876 & 2.276 & 1.400 & 160 \\
\hline Museu de la Música & 39 & 1.404 & 1.365 & 3.500 \\
\hline Museu Nacional de la Ciència i la Tècnica de & 9 & 1.148 & 1.139 & 12.656 \\
\hline Catalunya & 167 & 935 & 846 & 951 \\
\hline Museu de l'Institut Botànic de Barcelona & 996 & 629 & 377 \\
\hline Museu de Badalona & & & & \\
\hline
\end{tabular}




\begin{tabular}{|c|c|c|c|c|}
\hline Museo (ordenado por diferencia) & 2006 & 2009 & $\begin{array}{l}\text { Diferencia } \\
\text { 2006-2009 }\end{array}$ & $\begin{array}{c}\% \\
\text { Diferencia } \\
\text { 2006-2009 }\end{array}$ \\
\hline Museu Municipal Josep Aragay (Breda) & 6 & 526 & 520 & 8667 \\
\hline Museu de l'Empordà & 240 & 591 & 351 & 146 \\
\hline Museu d'Arqueologia de Catalunya & 0 & 345 & 345 & - \\
\hline Museu Episcopal de Vic & 124 & 468 & 344 & 277 \\
\hline Museu Tèxtil i d'Indumentària & 234 & 527 & 293 & 125 \\
\hline Museu Frederic Marès & 393 & 684 & 291 & 74 \\
\hline Museu del Cinema (Girona) & 233 & 392 & 159 & 68 \\
\hline Museu d'Art Jaume Morera (Lleida) & 212 & 336 & 124 & 58 \\
\hline Museu de la Pesca de Palamós & 8 & 132 & 124 & 1.550 \\
\hline Museu de Cardedeu & 7 & 107 & 100 & 1.429 \\
\hline Museu de Gavà & 0 & 87 & 87 & - \\
\hline Museu d'Art Modern de Tarragona & 10 & 88 & 78 & 780 \\
\hline Museu Geològic del Seminari de Barcelona & 29 & 101 & 72 & 248 \\
\hline Fundació Tàpies & 10 & 75 & 65 & 650 \\
\hline Museu Salvador Vilaseca (Reus) & 56 & 101 & 45 & 80 \\
\hline Museu de la Noguera & 22 & 61 & 39 & 177 \\
\hline Museu de Montserrat & 8 & 39 & 31 & 388 \\
\hline Museu Monestir de Pedralbes & 12 & 39 & 27 & 225 \\
\hline Museu Torre Balldovina & 53 & 80 & 27 & 51 \\
\hline Museu d'Art de Girona & 3 & 22 & 19 & 633 \\
\hline Museu de Ripoll & 114 & 132 & 18 & 16 \\
\hline Museu d'Història de Girona & 75 & 92 & 17 & 23 \\
\hline Museu Municipal de Nàutica del Masnou & 1 & 17 & 16 & 1.600 \\
\hline Museu de Terrassa & 28 & 37 & 9 & 32 \\
\hline Museu de Cervera & 5 & 13 & 8 & 160 \\
\hline Museu del Suro de Palafrugell & 7 & 15 & 8 & 114 \\
\hline Museu Molí Paperer de Capellades & 13 & 21 & 8 & 62 \\
\hline Museu Pau Casals & 5 & 13 & 8 & 160 \\
\hline Museu d'Història de Sabadell & 0 & 6 & 6 & - \\
\hline Centre d'Interpretació del Parc Güell & 0 & 5 & 5 & - \\
\hline Museu Agrícola de Cambrils & 0 & 4 & 4 & - \\
\hline L'Enrajolada Casa - Museu Santacana (Martorell) & 0 & 3 & 3 & - \\
\hline Museu Municipal Vicenç Ros (Martorell) & 0 & 3 & 3 & - \\
\hline Museu Deu (Vendrell) & 0 & 0 & 0 & 0 \\
\hline
\end{tabular}




\begin{tabular}{|c|c|c|c|c|}
\hline Museo (ordenado por diferencia) & 2006 & 2009 & $\begin{array}{c}\text { Diferencia } \\
2006-2009\end{array}$ & $\begin{array}{c}\% \\
\text { Diferencia } \\
2006-2009\end{array}$ \\
\hline Museu Etnològic del Montseny & 0 & 0 & 0 & 0 \\
\hline Museu Marítim de Barcelona & 0 & 0 & 0 & 0 \\
\hline Museu Municipal de Tossa de Mar & 42 & 40 & -2 & -5 \\
\hline Museu del Vidre de Vimbodí & 5 & 1 & -4 & -80 \\
\hline Museu de Zoologia & 12 & 7 & -5 & -42 \\
\hline Museu d'Història de Cambrils & 57 & 34 & -23 & -40 \\
\hline Museu Diocesà d'Urgell & 84 & 53 & -31 & -37 \\
\hline Catedral de Girona & 70 & 1 & -69 & -99 \\
\hline Museu del Joguet de Catalunya & 595 & 513 & -82 & -14 \\
\hline Museu del Futbol Club Barcelona & 174 & 8 & -166 & -95 \\
\hline Museu de la Pell d'Igualada & 170 & 1 & -169 & -99 \\
\hline Museu-Arxiu Municipal de Calella & 193 & 13 & -180 & -93 \\
\hline Biblioteca Museu Víctor Balaguer & 2.190 & 178 & -2.012 & -92 \\
\hline Fundació Miró & 10.500 & 2.229 & -8.271 & -79 \\
\hline
\end{tabular}

